\title{
Mechanistic behaviour and modelling of creep in powder metallurgy FGH96 nickel superalloy
}

\author{
Zichao Peng ${ }^{1 *}$, Gaofeng Tian ${ }^{1}$, Jun Jiang ${ }^{2}$, Muzi $L^{2}{ }^{2}$, \\ Yang Chen ${ }^{1}$, Jinwen Zou ${ }^{1}$, Fionn P.E. Dunne ${ }^{2^{*}}$
}

1. Science and Technology on Advanced High Temperature Structural Materials Laboratory, AVIC Beijing Institute of Aeronautical Materials, Beijing, 100095, China

2. Department of Materials, Royal school of Mines, Imperial College London, Exhibition Road, SW7 2AZ, UK

\begin{abstract}
The creep properties of a nickel-based superalloy at $700^{\circ} \mathrm{C}$ and $690 \mathrm{MPa}$ resulting from differing aging heat treatments have been investigated. The heat treatments gave rise to significantly different tertiary $\gamma^{\prime}$ precipitate distributions which in turn influence the propensity for precipitate shearing. The creep life was found to decrease with an increase of volume fraction of tertiary $\gamma^{\prime}$. It is shown that the $\gamma^{\prime}$ precipitates undergo shearing by matrix dislocations resulting in residual stacking faults which have been identified from TEM studies.
\end{abstract}

A physically-based crystal slip model for creep deformation in FGH96 has been developed. A critical $\gamma^{\prime}$ precipitate size is found to exist above which precipitate shearing occurs by strongly-coupled dislocations pairs. The critical size for FGH96 superalloy is less than $15 \mathrm{~nm}$, which is smaller than most of the $\gamma^{\prime}$ precipitates in the aged treated samples. A bimodal precipitate hardening model has been presented from which the slip strength resulting from differing heat treatments may be determined. Creep strain rate is found to decrease with increasing slip strength. The crystal slip model successfully captures the effect of heat treatment (in terms of $\gamma^{\prime}$ precipitate distributions) on resulting creep behavior in alloy FGH96.

\section{Introduction}

Powder metallurgy (PM) Ni-based superalloys are of the most important materials for high-temperature structural applications in turbine discs. Due to their good creep and tensile properties, and micro-structural stability up to $650^{\circ} \mathrm{C}$ for extended periods of exposure, the FGH96 superalloy is widely used in turbine discs ${ }^{[1,2]}$. Ni-based superalloy is solution strengthened by $\gamma^{\prime}\left(\mathrm{LI}_{2}\right.$ structure) precipitates which are uniformly distributed in a disordered FCC matrix ${ }^{[3]}$.

The creep behavior of PM superalloy is greatly dependent on the $\gamma$ ' precipitates, and so many investigators study the relationship between the creep properties and $\gamma$ ' precipitates by changing the creep conditions or solution heat-treatment. For example, Sugui $^{[4]}$ studied the microstructure and creep behaviuor of FGH95 superalloy through different solution heat-treatments. $\mathrm{Xie}^{[5]}$ addressed the creep behavior and role of dislocation networks in powder metallurgy (PM) superalloy.

Most investigations have been focused on the role of secondary $\gamma^{\prime}$ precipitates in 
terms of the creep behaviours of these alloys, but the tertiary $\gamma^{\prime}$ precipitates have seldom been carefully addressed ${ }^{[6]}$. However, Viswanathan investigated the creep deformation mechanisms at intermediate temperatures in Rene 88DT superalloy, and the results show that for the finer microstructure, anti-phase boundaries (APBs) must be created in the tertiary $\gamma^{\prime}$ particles, while for the coarser microstructure, stacking faults (SFs) are created in the tertiary $\gamma^{\prime}$ precipitates $^{[7]}$. Locq studied the role of tertiary $\gamma^{\prime}$ precipitates in creep behavior at $700^{\circ} \mathrm{Cof}$ PM NR3 superalloy and found that the presence of fine tertiary $\gamma^{\prime}$ precipitates in the $\gamma$ matrix between the coarser secondary $\gamma^{\prime}$ precipitates impeded spreading of a/2<110> dislocations through the $\gamma-\gamma$ ' microstructure. It is therefore apparent that the tertiary $\gamma^{\prime}$ precipitates potentially play a significant role in influencing and controlling the creep properties of Ni-based superalloy and this is the focus of the present study.

We utilize differing long-term ageing methods to obtain superalloys with various $\gamma$ ' precipitate distributions, which are then tested under the same creep loading and temperature conditions. The results obtained provide insight into the strengthening effects of $\gamma^{\prime}$ precipitates in creep, and in addition provide a mechanistic modelling methodology to predict resulting creep behavior so that the long-term performance of key aero-engine components such as turbine discs may be obtained.

Four different heat treatments have been designed for the FGH96 superalloy in order to obtain various alloy microstructures with differing $\gamma^{\prime}$ precipitate distributions, but importantly with same grain morphology and texture. Creep tests were carried out on samples with the differing microstructures at the same stress and temperature. The interaction between the $\gamma^{\prime}$ precipitates and the matrix dislocations have been studied and the creep mechanisms established to enable a physically-based crystal plasticity model ${ }^{[8]}$ to be developed which explicitly accounts for $\gamma^{\prime}$ microstructural change, and its influence on the resulting creep properties of FGH96 superalloy.

\section{Experimental Methodology}

The gas atomization method was used for powder preparation, following which the powder particles were encapsulated in stainless steel and sealed after vacuum degassing. After hot isostatic pressing (HIP), the billet was forged to pancake, which was followed with standard heat treatment $\left(1150^{\circ} \mathrm{C}\right.$ solution heat treatment + two stages of aging treatment). After heat treatment, the pancake was divided into four parts. In order to obtain different $\gamma$ ' distributions, the alloy parts were processed with four different aging treatments which are detailed in Table 1. The creep samples were machined from equivalent locations of each pancake part from the rim region.

Table 1 Aging treatment parameters

\begin{tabular}{c|c}
\hline & Aging treatment \\
\hline HT1 & $800^{\circ} \mathrm{C} / 500 \mathrm{~h}$, Air cooling \\
\hline HT2 & $700^{\circ} \mathrm{C} / 1000 \mathrm{~h}$, Air cooling \\
\hline HT3 & $700^{\circ} \mathrm{C} / 3000 \mathrm{~h}$, Air cooling \\
\hline HT4 & $750^{\circ} \mathrm{C} / 1000 \mathrm{~h}$, Air cooling \\
\hline
\end{tabular}


The sample grain size and textures were investigated before creep testing by optical microscopy (OM) and electron backscattered diffraction (EBSD), respectively. The $\gamma^{\prime}$ distributions produced from the four aging treatments are examined with secondary emission scanning electron microscopy (SEM: LEO Gemini 1525). For the SEM, the samples were ground, polished and electro-etched at $2.5 \mathrm{~V}, 2 \mathrm{~A}$ for one second at room temperature.

The precipitate distribution functions were obtained from SEM images using ImageJ software to produce binary $\gamma$ and $\gamma^{\prime}$ images ${ }^{[9]}$. By setting appropriate contrast thresholds, individual precipitate areas were reliably determined, allowing equivalent precipitate radius and area fraction to be calculated. In order to carry out quantitative analysis, more than five images were randomly selected and analyzed for each creep sample. In this work, we equate the area fraction with the volume fraction of the $\gamma$ ' precipitates.

Thin foils for transmission electron microscopy (TEM) observations were prepared from discs sectioned $\sim 45^{\circ}$ to the stress axis of the creep-tested samples ${ }^{[10]}$. The foils were prepared by the twin-jet thinning technique using a solution consisting of $10 \%$ $\mathrm{HClO}_{4}+45 \%$ 1-Butonal $+45 \% \mathrm{CH}_{3} \mathrm{COOH}$ at $-45^{\circ} \mathrm{C}$. Observations of the deformation structures were conducted using a JEOL JEM 2100F operated at $200 \mathrm{kV}$.

\section{Results}

\subsection{Microstructure characterization}

Fig. 1 shows the pole figures (PFs) obtained for the FGH96 superalloy before creep testing; more than 200 grains were analyzed. The alloy samples had minimal preferred crystallographic orientation (texture) before creep testing, so that texture is not deemed to be important in determining the resulting creep properties of the superalloy in our study.

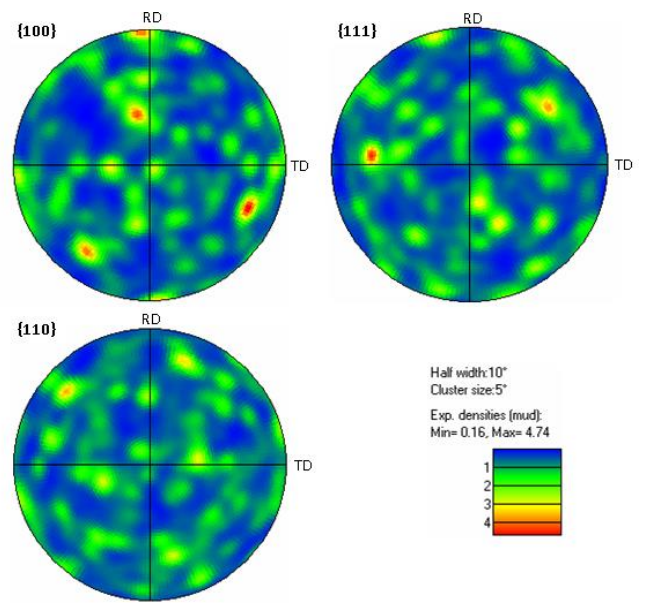

Fig.1 showing the $\{100\},\{111\}$ and $\{110\}$ pole figures of the FGH96 superalloy before creep testing

Before creep testing, the grain size of the alloy samples with different aging-treatments were observed by OM shown in Fig. 2, and all the samples have a similar grain size, which is about $20 \mu \mathrm{m}$. As for crystallographic orientation, the grain 
size and grain morphology were deliberately kept constant for all samples such that these factors do not influence the creep properties resulting from different aging-treatments.

In general, creep properties of Ni-based superalloys are mainly influenced by grain size, texture, test conditions and $\gamma^{\prime}$ distributions. In this work, both the grain size and texture are similar for all the creep samples, and the same creep test conditions are imposed. Therefore, the $\gamma^{\prime}$ distribution becomes the single key factor influencing the differing creep properties of FGH96 superalloy in these tests.
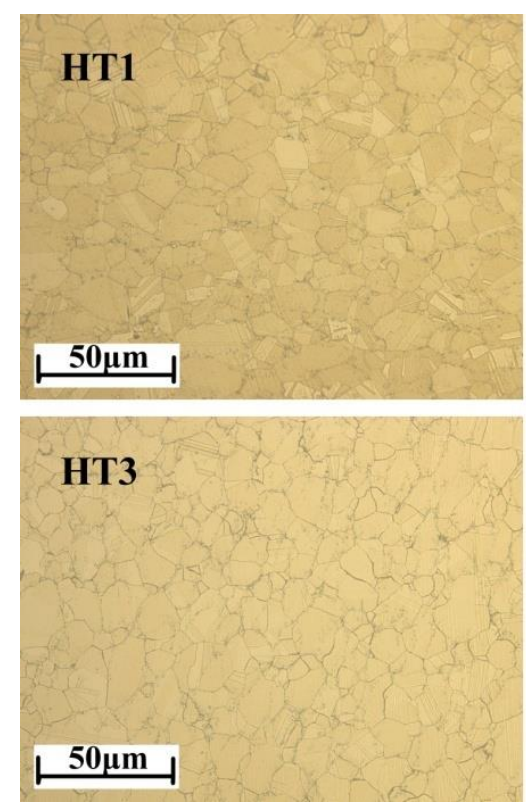
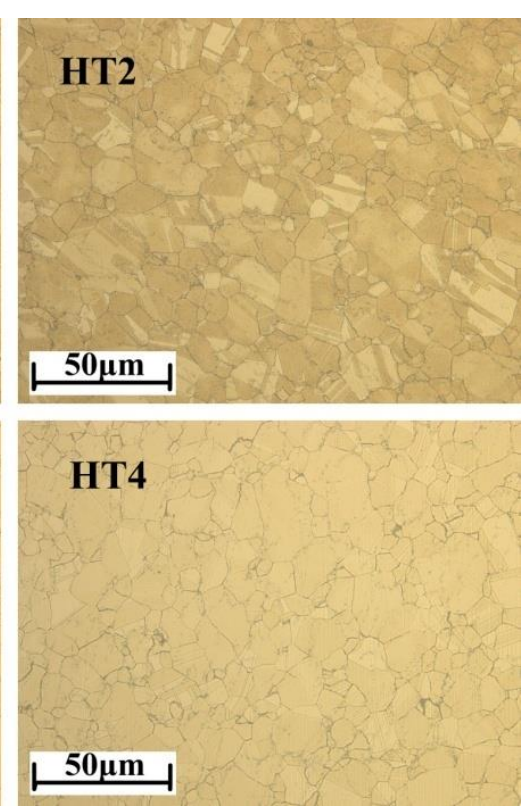

Fig. 2 The grain size of FGH96 with different aging treatments

The $\gamma^{\prime}$ distribution images are shown in Figure 3. Due to different aging-treatments, the $\gamma^{\prime}$ distributions are very distinctive. As the solution heat treatment is super-solvus, there is no primary $\gamma^{\prime}$ in the superalloy samples. In the HT1 and HT2 samples, tertiary $\gamma^{\prime}$ precipitates barely exist; otherwise, the volume fraction of tertiary $\gamma$ ' is much larger in HT3 and HT4. A summary of the microstructural statistics calculated related to the $\gamma^{\prime}$ distributions is shown in Table. 2. 

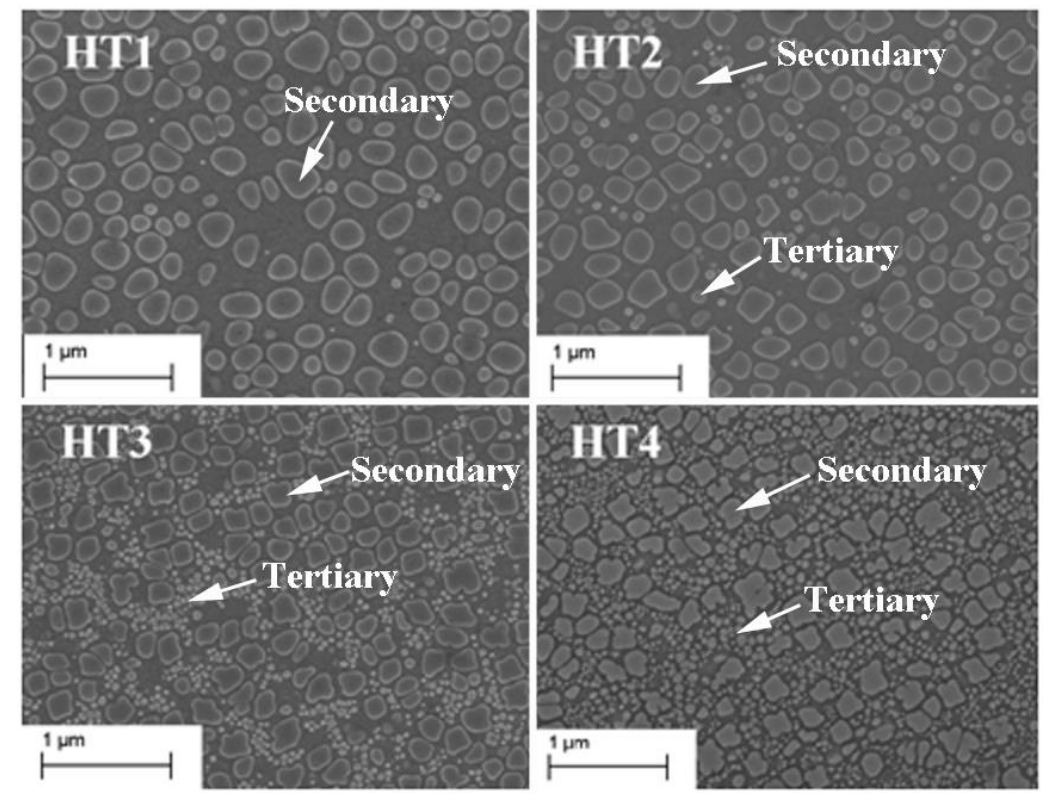

Fig.3The $\gamma^{\prime}$ distribution images of the superalloy resulting from different aging treatment

Table 2. Summary of $\gamma^{\prime}$ distribution statistics

\begin{tabular}{c|c|c|c|c|c}
\hline $\begin{array}{c}\text { Sample } \\
\text { No }\end{array}$ & $\begin{array}{c}\text { Average radius } \\
\text { of secondary } \gamma^{\prime} \\
\text { phase, } r_{s}(\mathrm{~nm})\end{array}$ & $\begin{array}{c}\text { Volume } \\
\text { fraction of } \\
\text { secondary } \gamma^{\prime} \\
\text { phase, } f_{s}(\%)\end{array}$ & $\begin{array}{c}\text { Average } \\
\text { radius of } \\
\text { tertiary } \gamma^{\prime} \\
\text { phase, } r_{t}(\mathrm{~nm})\end{array}$ & $\begin{array}{c}\text { Volume fraction } \\
\text { of tertiary } \gamma^{\prime} \\
\text { phase, } f_{t}(\%)\end{array}$ & $\begin{array}{c}\text { Volume } \\
\text { fraction of } \gamma \\
\text { phase, } f(\%)\end{array}$ \\
\hline HT1 & 102.81 & 44.62 & 32.99 & 0.77 & 45.39 \\
\hline HT2 & 96.27 & 46.13 & 31.23 & 2.67 & 48.79 \\
\hline HT3 & 86.82 & 37.96 & 22.99 & 11.64 & 49.60 \\
\hline HT4 & 85.13 & 29.52 & 22.37 & 20.19 & 49.71 \\
\hline
\end{tabular}

\subsection{Creep tests results}

The creep test matrix is shown in Table 3. All creep tests were performed under constant load conditions. There are two groups of creep tests in this study. Group 1 comprises the samples with different aging-treatments, namely with different $\gamma$ ' distributions, which were all tested at $690 \mathrm{MPa}$ and $700^{\circ} \mathrm{C}$ (each aging treatment with two samples). In group 2, three further creep tests were performed on identical bimodal samples with standard heat-treatment at $700^{\circ} \mathrm{C}$, but the stresses are $750 \mathrm{MPa}$, $720 \mathrm{MPa}$ and $660 \mathrm{MPa}$, respectively. The group 2 creep tests were used to calculate the thermodynamic parameters in the crystal plasticity model ${ }^{[11]}$ described later. The creep life and secondary creep rate are also shown in Table 3 , and all the creep tests were terminated once a strain level of $3 \%$ had been achieved. 
Table 3 Creep test matrix and results

\begin{tabular}{|c|c|c|c|c|}
\hline Group & Heat treatment & $\begin{array}{c}\text { Creep } \\
\text { condition }\end{array}$ & Creep life, $\mathrm{t}(\mathrm{h})$ & $\begin{array}{c}\text { Secondary creep } \\
\text { rate, } \mathrm{s}^{-1}\end{array}$ \\
\hline \multirow{4}{*}{ Group1 } & HT1 & \multirow{4}{*}{$\begin{array}{c}690 \mathrm{MPa} \\
700^{\circ} \mathrm{C}\end{array}$} & 20.1 & $3.52 \times 10^{-5}$ \\
\hline & HT2 & & 39.5 & $2.15 \times 10^{-5}$ \\
\hline & HT3 & & 225.33 & $8.08 \times 10^{-7}$ \\
\hline & HT4 & & 394.83 & $5.55 \times 10^{-7}$ \\
\hline \multirow{3}{*}{ Group2 } & \multirow{3}{*}{$\begin{array}{c}\text { Standard heat } \\
\text { treatment }\end{array}$} & $750 \mathrm{MPa}, 700^{\circ} \mathrm{C}$ & 134 & $9.69 \times 10^{-9}$ \\
\hline & & $720 \mathrm{MPa}, 700^{\circ} \mathrm{C}$ & 180 & $7.02 \times 10^{-9}$ \\
\hline & & $660 \mathrm{MPa}, 700^{\circ} \mathrm{C}$ & 490 & $3.83 \times 10^{-9}$ \\
\hline
\end{tabular}

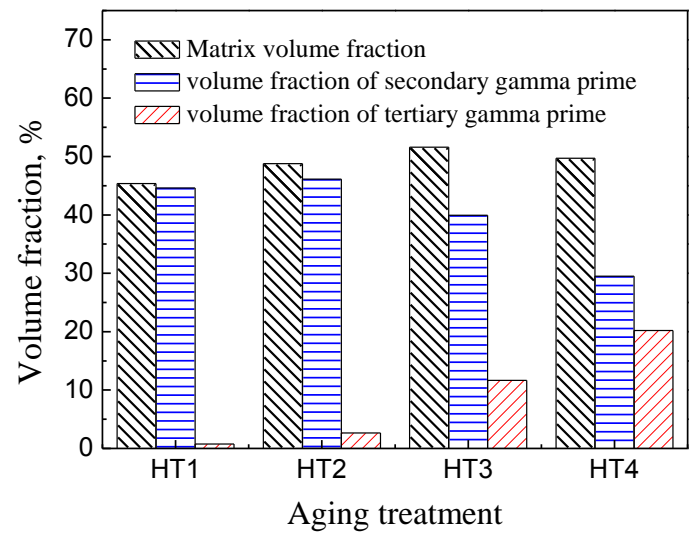

Fig. 4 The area (volume) fractions of gamma prime in the superalloy with different aging treatments

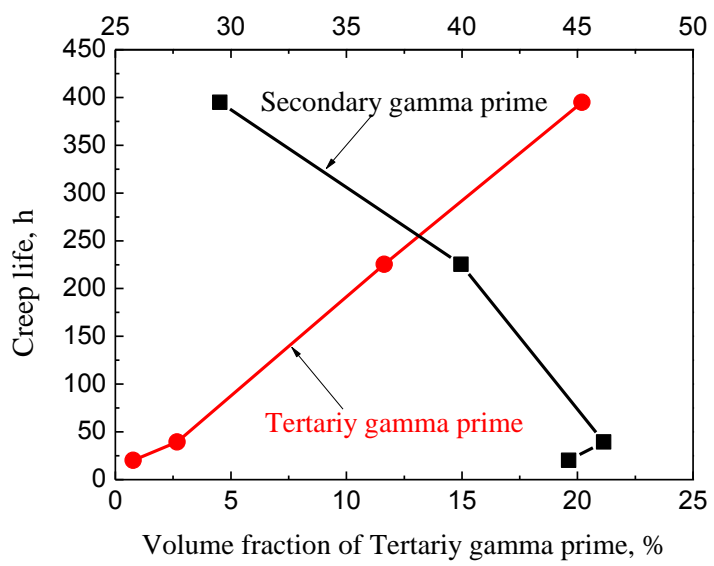

Fig. 5 Relationship between the volume fraction and the creep life (ie the time to achieve a strain of $3 \%$ )

From Table 2, it is observed that the range of radius is about the same for both groups across the heat treatments. Fig. 4 shows the variation of the $\gamma^{\prime}$ precipitate volume fraction (including secondary, tertiary and matrix volume fraction) in the superalloy with different aging treatments. Although the matrix volume fraction of $\gamma^{\prime}$ precipitates changes little, the volume fraction of tertiary $\gamma^{\prime}$ varies significantly. From Figure 5, it is also shown that the creep life (ie the time to achieve a strain of 3\%) 
increases with the increase of volume fraction of tertiary $\gamma^{\prime}$ precipitates, which indicates that the strengthening effect of tertiary $\gamma^{\prime}$ is markedly different to that of the secondary $\gamma^{\prime}$. This is of interest since most previous studies primarily focus on the role of the secondary $\gamma^{\prime}$ phase.

\subsection{Creep Mechanisms}

PM nickel-base superalloys are precipitation hardening alloys such that the $\gamma^{\prime}$ particles act to impede the motion of glissile dislocations. The shearing and the Orowan looping models are the two predominant mechanistic explanations which describe the interaction between the mobile dislocations and the precipitates ${ }^{[12-13]}$. In particular, the transition from cutting of $\gamma^{\prime}$ precipitates by paired dislocations to single dislocation Orowan looping occurs at large (over-aged) spherical precipitates ${ }^{[14]}$.

For our creep samples, the distribution and form of the dislocations is observed through TEM, and the images obtained are shown in Fig. 6 and Fig. 7. In Fig.6, a large number of matrix dislocations locate near to a grain boundary, suggesting that the matrix dislocations originate at the grain boundary. In Fig.7, there are many stacking faults observed in the secondary $\gamma^{\prime}$ precipitate, which are induced by the matrix dislocation $1 / 2 \mathrm{a}<110>$. Matrix dislocations $(1 / 2 \mathrm{a}<110>)$ firstly glide in the $\gamma$ matrix, and then interact with the $\gamma^{\prime}$ precipitates, resulting in dissociation into two partial dislocations, leaving stacking faults in the $\gamma^{\prime}$ precipitate ${ }^{[15]}$.

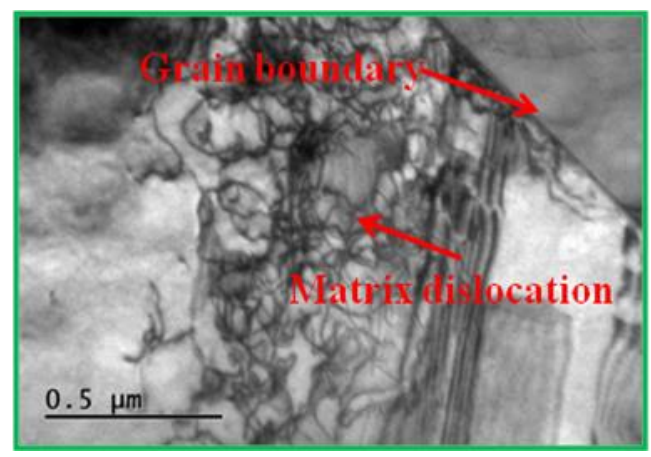

Fig. 6 Matrix dislocations close to a grain boundary

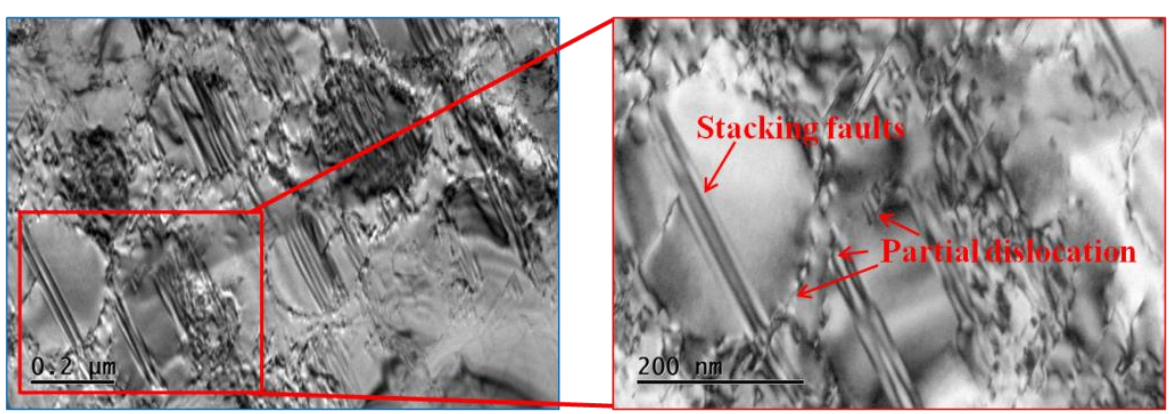

Fig. 7 TEM images within the creep samples

All the creep tested samples with differing aging treatments were analyzed by TEM, and stacking faults of the type described were observed in all of them. It is concluded 
that the $\gamma^{\prime}$ precipitates were sheared by the matrix dislocations in all the creep samples, which indicates that the interaction between dislocations and precipitates is controlled by the shearing model, as opposed to the Orowan looping process.

\section{Mechanistic creep model}

\subsection{Precipitate hardening mechanism}

The critical resolved shear stress (CRSS), or intrinsic slip system strength, may be estimated by consideration of the dislocation interaction mechanisms ${ }^{[16]}$. Based on pair-wise dislocation motion, Huther and Reppich proposed a modified hardening model which led to some new aspects in respect of the strength of hardening in the case of strong paired coupling at larger particle sizes ${ }^{[17]}$.

The critical resolved shear stress is estimated which is necessary to move two coupled dislocations in the $\langle 110\rangle$ direction on the $\{111\}$ plane cutting through $\gamma$ ' precipitates. The first model describes the CRSS for two weakly coupled dislocations to shear through ordered precipitates in a disordered matrix. The second describes the same motion for strongly coupled dislocations.

For our samples, all the precipitates are assumed to be spherical. For small particles, the CRSS $\tau_{c}$ is determined by the stress necessary to move weakly coupled dislocation pairs as follows ${ }^{[18]}$

$$
\tau_{c}=\frac{\gamma_{A P B}}{2 b}\left[\left(\frac{6 \gamma_{A P B} f r}{\pi T}\right)^{1 / 2}-f\right]
$$

where $\gamma_{A P B}$ is the APB energy, $\sim 0.279 \mathrm{~J} / \mathrm{m}^{2}$ at $700^{\circ} \mathrm{C}^{[19]}, f$ is the volume fraction of $\gamma^{\prime}$ phase, and $r$ is the average radius of the precipitate. The constant line tension $T$ may be estimated according to ${ }^{[20]}$

$$
T=\frac{G b^{2}}{2}
$$

where $G$ is the isotropic elastic shear modulus, which can be obtained from the known Young's Modulus, E (199.8Gpa) and Poisson's ratio, $\mu$ (0.352) of FGH96 and taking

the Burgers vector magnitude, $b$, to be equal to $\sqrt{2} a / 2$ for $\{111\}$ slip, in which $a$ is the lattice parameter, taken to be $0.359 \mathrm{~nm}$ for FGH96 and obtained from XRD. Assuming isotropic linear elasticity the shear modulus is obtained from

$$
\mathrm{G}=\frac{\mathrm{E}}{2(1+\mu)} .
$$

For larger spherical particles, where dislocations cut by forming strongly coupled pairs, and the CRSS is given by ${ }^{[18]}$ 


$$
\tau_{c}=\sqrt{\frac{3}{2}}\left(\frac{G b}{r}\right) f^{\frac{1}{2}}\left(\frac{\omega}{\pi^{3 / 2}}\right)\left(\frac{2 \pi \gamma_{A P B^{r}}}{\omega G b^{2}}-1\right)^{1 / 2}
$$

where $\omega$ is a constant which accounts for the elastic repulsion between the strongly paired dislocations and which is of the order of unity.

Combining equations (1) and (4), the CRSS can be calculated with the given volume fraction and $\gamma^{\prime}$ precipitate radius. Fig. 8 shows the resulting CRSS versus precipitate radius for the two dislocation slip mechanisms. The volume fraction $f$ is taken as $0.05,0.25$ and 0.5 for the three cases shown

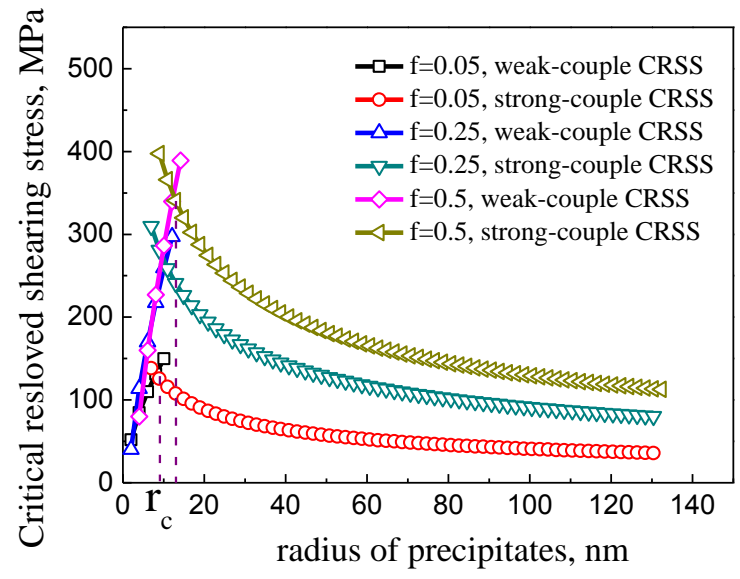

Fig. 8 Theoretical critical resolved shear stress versus precipitate radius relationships for the two dislocation motion mechanisms at $700^{\circ} \mathrm{C}$

From Fig.8, it is seen that for a given $\gamma^{\prime}$ volume fraction, there is an intersection between the two mechanism curves. At this point, the CRSS from the two models are equal, and the particle radius at this point of intersection is defined as the critical precipitate radius $r_{c}$, such that when precipitate radius $r<r_{c}$, the dislocation movement is mainly controlled by the weakly-coupled model, and conversely when $r>r_{c}$, the mechanism is such that the strongly-coupled model dominates. For the FGH96 superalloys with different aging treatments, the critical radii $r_{c}$ are less than $20 \mathrm{~nm}$. All the secondary and tertiary $\gamma^{\prime}$ precipitate radii in our creep samples are larger than 20nm, as shown in Fig.9, such that the strong-coupled dislocation pair model controls the dislocation movement in all the creep samples.
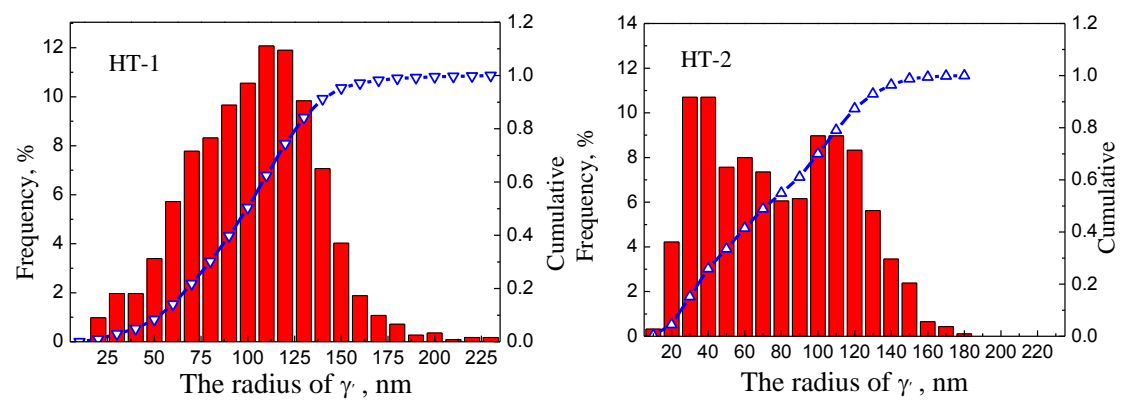

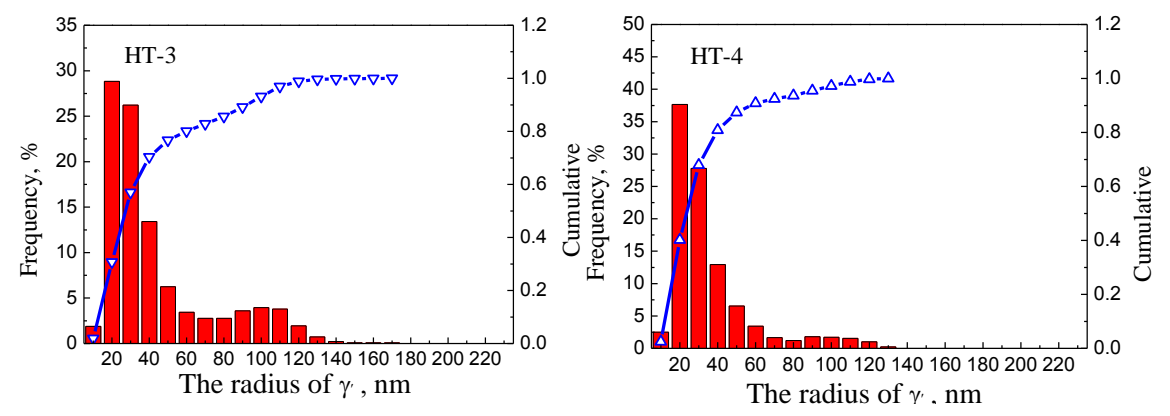

Fig. 9 Precipitate size histograms for different sample aging treatment

\subsection{Hardening by bimodal precipitate dispersions}

Through control of aging treatments, it is possible to precipitate two classes of particles in the creep samples considered: $f_{s}$ and $r_{s}$ are the volume fraction and the average radius of the secondary $\gamma^{\prime}$ precipitates, and $f_{t}$ and $r_{t}$ are the volume fraction and the average radius of the tertiary $\gamma^{\prime}$ precipitates. The secondary and tertiary $\gamma^{\prime}$ precipitates are often deemed to operate independently when considering their inhibition of dislocation gliding. In the literature, at least three different suggestions for the critical shearing stress function for bimodal precipitates dispersions exist ${ }^{[13]}$. To simplify, and in the absence of definitive evidence, we assume that the total critical shearing stress for the FGH96 superalloy is given by a combination of both secondary and tertiary strengths as

$$
\tau_{c}=\left(\tau_{s}^{2}+\tau_{t}^{2}\right)^{1 / 2}
$$

where $\tau_{s}$ and $\tau_{t}$ are the critical shear stresses for the secondary and tertiary $\gamma^{\prime}$ precipitates, respectively.

Combining equations (1) (5), with the precipitate properties given in Table 1, we can estimate the critical resolved shear stress for each heat treated creep sample material, and the calculated results are shown in Fig. 10. As shown in this figure, the critical shearing stress increases with the increasing volume fraction of tertiary $\gamma^{\prime}$ precipitates. However, as the volume fraction of tertiary $\gamma$ ' precipitate increases, so the secondary creep strain rate decreases. This indicates that the secondary creep strain rate is controlled by the critical shearing stress.Therefore, in order to improve the creep properties of precipitate-hardened alloys, the results suggest that increasing the critical resolved shear stress is beneficial. 


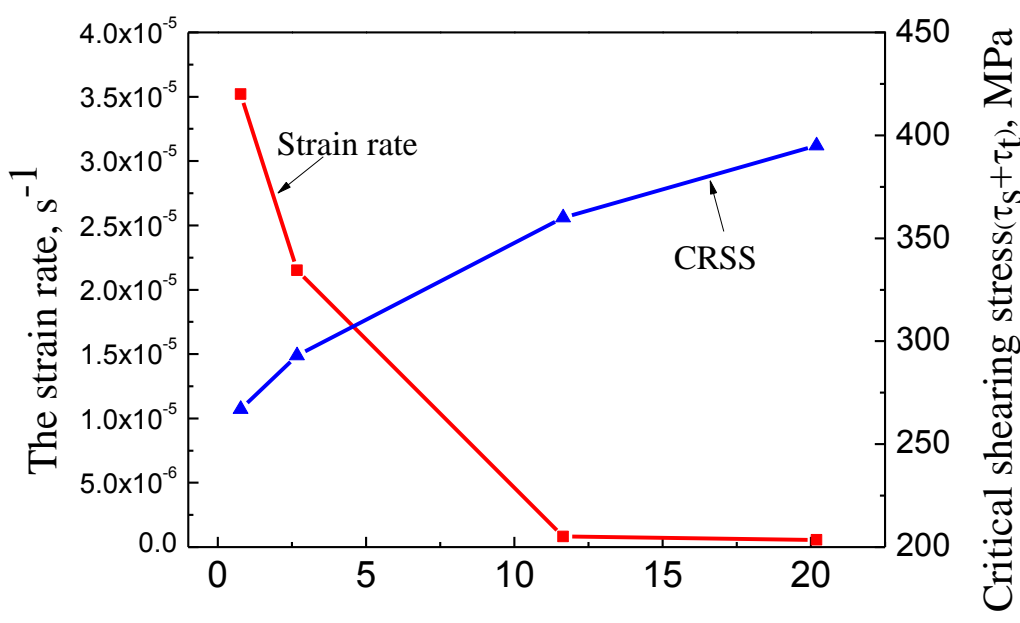

The Volume fraction of tertiary $\gamma^{\prime}\left(f_{t}\right), \%$

Fig. 10 Relationships between strain rate, critical shearing stress and volume fraction of tertiary $\gamma^{\prime}$ phase

\subsection{Physically-based creep model}

Gibbs developed a rate equation for dislocation movement from first principles using a statistical-thermodynamic analysis of dislocation glide controlled by dispersed local obstacles ${ }^{[11,20]}$. The creep strain rate, $\dot{\gamma}$, is determined from the Orowan equation as

$$
\dot{\gamma}=\rho_{g} v_{g} b
$$

in which $\rho_{g}$ is the density of gliding dislocations with average velocity $v_{g}$, and $b$ is Burger's vector magnitude. Obstacles such as impurity atoms, forest dislocations and precipitates, pin gliding dislocations requiring a local thermal activation event to enable the dislocations to overcome the energy barrier and escape. The product of the length of the thermal activation event, $d$, and the rate of escape, $\Gamma\left(\mathrm{s}^{-1}\right)$, of pinned dislocations gives rise to the average velocity. The rate of escape was calculated statistically by Granato et al ${ }^{[21]}$, to give

$$
\Gamma=\frac{v b}{2 l} \exp \left(-\frac{\Delta G}{k_{b} T}\right)
$$

in which $v$ is the frequency of dislocations attempting to jump across the energy barriers, $l$ the pinned dislocation segment length, $k_{b}$ the Boltzman constant, and $T$ is the absolute temperature. The velocity was then determined as

$$
v_{g}=\frac{A}{l} \Gamma=\frac{A b v}{2 l^{2}} \exp \left(-\frac{\Delta G}{k_{b} T}\right)
$$

in which $A$ is the area swept out by a dislocation segment released during thermal activation, and $\Delta G$ is the Gibbs free energy which, in the presence of a stress field, 
becomes

$$
\Delta G=\Delta F-\tau_{e} \Delta V
$$

in which $\Delta F$ is the Helmholtz free energy, and $\tau_{e} \Delta V$ is the work carried out by the stress field, in which $\tau_{\varepsilon}$ is the effective stress, and $\Delta V$ is the activation volume. Substituting equation (9) into (8) then gives the shear strain rate as

$$
\dot{\gamma}=\frac{\rho_{g} A b^{2} v}{2 l^{2}} \exp \left(-\frac{\Delta F}{k_{b} T}\right) \exp \left(\frac{\tau_{e} \Delta V}{k_{b} T}\right)
$$

If both forward and backward activation events are accounted for, in a symmetrical arrangement, and the area swept out by a dislocation segment $A$ is estimated as $l^{2}$, then equation (10) takes the form

$$
\dot{\gamma}=\rho_{g} b^{2} v \exp \left(-\frac{\Delta F}{k_{b} T}\right) \sinh \left(\frac{\tau_{e} \Delta V}{k_{b} T}\right)
$$

In equation (11), the density of gliding dislocations $\rho_{g}$, the Burger's vector $b$, the frequency of dislocations attempting to jump $v$, Boltzman constant $k_{b}$, the temperature $T$, the Helmholtz free energy $\Delta F$ and the activation volume $\Delta V$ are known or may be estimated from creep data. Therefore, the effective stress is the only independent variable influencing the creep strain rate, and may be expressed as the difference between the applied stress and the intrinsic slip system strength as

$$
\tau_{e}=\tau-\tau_{c}
$$

For the simple case of purely uniaxial stressing, the resolved shear stress on a given slip system may be determined from Schmid rule as

$$
\tau=\sigma \cos \theta \cos \varphi
$$

in which $\sigma$ is the applied stress, $\theta$ the angle between the loading direction and slip direction, and $\varphi$ is the angle between the loading direction and slip plane normal. Substituting equation (12) and (13) into equation (11), then gives the creep strain rate as:

$$
\dot{\gamma}=\rho_{g} b^{2} v \exp \left(-\frac{\Delta F}{k_{b} T}\right) \sinh \left(\frac{\left(\sigma \cos \theta \cos \varphi-\tau_{c}\right) \Delta V}{k_{b} T}\right)
$$


When the precipitate distribution parameters are known (as they are in this study), the critical resolved shear stress can be calculated from equations $(1) \sim(5)$, such that with knowledge of the applied uniaxial stress, the secondary creep strain rate may be calculated from equation (14).

\subsection{Assessment of the crystal model}

In order to assess the physical basis of the model presented, it is necessary to determine the Helmholtz free energy $\Delta F$ and the activation volume $\Delta V$ from creep experiments. The secondary and tertiary particle radii and volume fractions are given in Table 2. The Burger's vector magnitude for our superalloy is taken as $0.254 \mathrm{~nm}$, and the density of mobile dislocations is estimated to be $10^{10} \mathrm{~m}^{-2[22]}$, and the required parameters are summarized in Table. 4. It remains to determine the rate-controlling activation energy and activation volume.

Table 4 parameters in creep model

\begin{tabular}{cc}
\hline$\rho_{g}$ & $1 \times 10^{10} \mathrm{~m}^{-2}$ \\
\hline$b$ & $0.254 \mathrm{~nm}$ \\
\hline$v$ & $1 \times 10^{11} \mathrm{~s}^{-1}$ \\
\hline$k_{b}$ & $1.381 \times 10^{-23} \mathrm{~J} / \mathrm{K}$ \\
\hline$T$ & $973 \mathrm{~K}$ \\
\hline
\end{tabular}

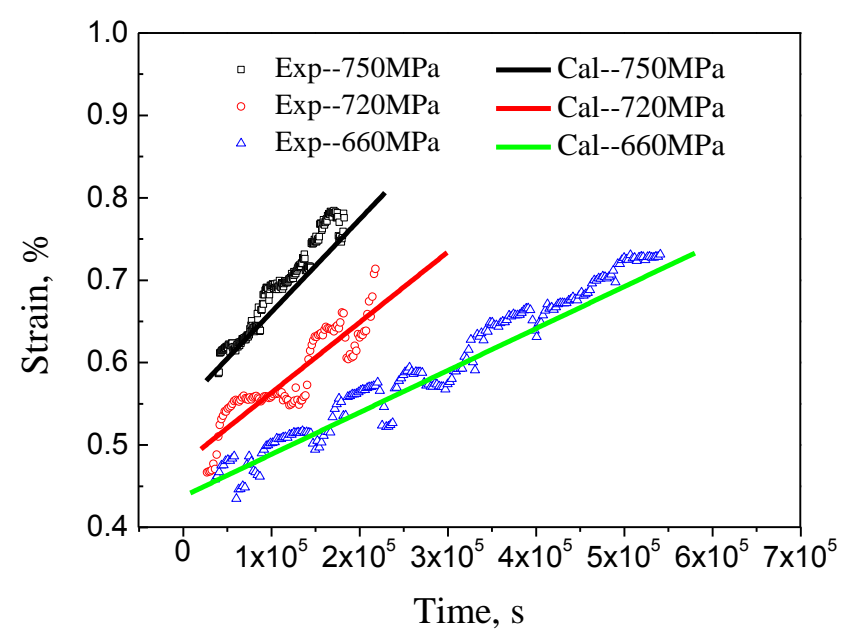

Fig. 11 Calculated and experimentally measured creep test behavior at $700^{\circ} \mathrm{C}$ for standard heat treated alloy FGH96 for the stresses shown 
Fig. 11 shows the calculated creep strain results under different stresses at $700^{\circ} \mathrm{C}$, obtained by calibrating the model to provide best correspondence with the experimental creep tests carried out on the standard heat treated samples, given in Table 3 (group 2). The Helmholtz free energy $\Delta F$ for FGH96 superalloy is determined to be $2.6509 \times 10^{-19} \mathrm{~J}$, and the activation volume $\Delta V$ to be $2.8 \times 10^{-23} \mathrm{~m}^{3}$. The model so determined is next utilized to assess the creep behavior of the samples which have undergone differing heat treatments, as detailed in Table 3 (group 1).

The critical resolved shear stresses for each of the differently heat treated FGH96 superalloys are obtained from the hardening model captured in equations (1), (4) and (5) and the corresponding calculated CRSSs $\tau_{c}$ are shown in Table. 5. Strong differences in the heat treated sample CRSSs, $\tau_{c}$, are observed, resulting from the slip resistance developed by the differing precipitate systems in heat treatments HT1 to HT4, which lead to the differing predicted creep strain rates shown, and compared with experimental measurements, in figure 12. The predicted creep strain behavior is obtained from equation (14).

Table 5 the calculated critical shear stress $\tau_{c}$

\begin{tabular}{ccccc}
\hline Heat treatment & HT1 & HT2 & HT3 & HT4 \\
\hline$\tau_{c}(\mathrm{MPa})$ & 123 & 132 & 177 & 200 \\
\hline
\end{tabular}

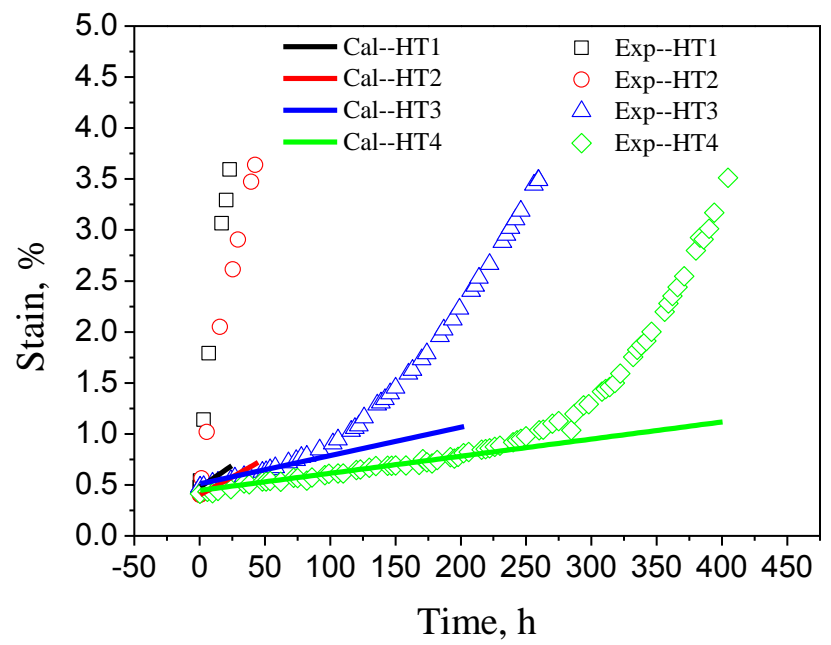

Fig. 12 Predicted and experimental creep response for superalloy FGH96 at $700^{\circ} \mathrm{C}$ for the heat treatments shown.

Figure 12 shows the predicted strain-time curves for secondary creep only, since the mechanisms leading to tertiary creep response observed in the experiments have not been addressed in this model. The predicted secondary creep of heat treated 
samples HT3 and HT4 show good agreement with the experimental results. However, differences are seen for samples HT1 and HT2 creep tests, largely resulting from the apparent negligible secondary creep developing in these experimental samples which, at the applied stress of $690 \mathrm{MPa}$ appear to undergo tertiary creep from the onset of loading.

The activation volume $\Delta V$ in the model is taken to be constant in this paper, but in reality is likely to be dependent on the $\gamma^{\prime}$ distribution. The model proposed by Manonukul et al ${ }^{[11]}$ indicates that there is a critical size of $\gamma^{\prime}$ precipitate, above which the activation volume is that relevant to dislocation bowing at precipitates, and can be determined by $\Delta V=l b^{2}$, in which $l$ is the spacing between $\gamma$ ' precipitates, and $b$ is the Burger vector magnitude. A critical activation volume $\Delta V_{c}$ for cutting was introduced depending on a critical size of $\gamma^{\prime}$. However, for the materials considered in this paper, precipitate shearing by matrix dislocations was found exclusively to occur (as opposed to bowing).

According to equations (4) and (5), the slip strengths $\tau_{s}$ and $\tau_{t}$ may be calculated with knowledge of the $\gamma^{\prime}$ distribution parameters independently, and matrix dislocation shearing likely determined by the larger one of the two. Therefore, there exists a critical volume fraction of tertiary $\gamma^{\prime}, f_{c}$, at which $\tau_{s}=\tau_{t}$. When $f_{\text {tertiary }}>f_{c}$, then $\tau_{t}>\tau_{s}$, and the matrix dislocation shearing is controlled by the tertiary $\gamma^{\prime}$, and the activation volume depends on the distribution of tertiary $\gamma^{\prime}$. For the converse scenario, the activation volume depends on the secondary $\gamma^{\prime}$ distribution. However, the relationship between the activation volume and the $\gamma^{\prime}$ distribution needs furthur investigation in order to provide better models.

\section{Conclusions}

Four different $\gamma^{\prime}$ distributions in FGH96 superalloy were produced by aging treatment. Prior to creep testing, the grain morphology and texture were investigated to ensure that the $\gamma^{\prime}$ distribution was the only varying factor influencing creep behavior. A number of identical creep tests were performed on differently aged samples and the results were assessed against a physically-based model developed in this work.

The $\gamma^{\prime}$ particle distributions within the creep samples were analyzed by SEM and Image $\mathbf{J}$ software. The differing aging treatments led to significant volume fraction changes of $\gamma^{\prime}$ precipitates. Inspection of the complete set of creep curves suggests that a larger volume fraction of tertiary $\gamma^{\prime}$ leads to a lower secondary creep rate, indicating that the strengthening effect of tertiary $\gamma^{\prime}$ is more marked than that for secondary $\gamma^{\prime}$. Through TEM characterization, many stacking faults and partial dislocations were observed in the creep samples. This suggests that at the given stress and temperature, the $\gamma$ ' precipitates were sheared by matrix dislocations leaving residual stacking faults.

The paper presents a physically-based crystal-based slip rule for creep deformation in alloy FGH96, in which the slip strength, or CRSS $\tau_{c}$, is found to be the most 
important factor influencing secondary creep rate, with strain rate decreasing with increasing $\tau_{c}$. The creep model proposed enables the effect of aging heat treatments in alloy FGH96 to be captured with reasonable agreement with experimental results.

\section{References}

[1] Nie L, Zhang L, Zhu Z, Xu W. Microstructure evolution modeling of FGH96 superalloy during inertia friction welding process, Finite Elements in Analysis and Design, 2014; 80:63.

[2] Yuli Gu, Yuhuai He, Shiyu Qu. Thermo-mechanical fatigue behavior of nickel-base powder metallurgy superalloy FGH96 under tension-tension loading, Acta Metallurgica SINICA, 2010; 3(2): 147

[3] Sims, C. T. , Stoloff, N. S., Hagel, W. C., Fundamentals of Strengthening in Superalloys II, Wiley-interscience, New York, NY, 1987; 61.

[4] TianSugui, Xie Jun, Zhou Xiaoming, Microstructure and creep behavior of FGH95 nickel-base superalloy, Materials Science and Engineering A, 2011; 528: 2076-2084.

[5] Jun Xie, SuguiTian, Li Juan Shang, Creep behaviors and role of dislocation network in a powder metallurgy Ni-based superalloy during medium-temperature, Materials Science and Engineering A, 2014, 606: 304-312.

[6] D. Locq, P. Caron, S. Raujol, On the role of tertiary $\gamma^{\prime}$ precipitates in the creep behavior at $700^{\circ} \mathrm{C}$ of a PM disk superalloy, Superalloys 2004: 179.

[7] G. B. Viswanathan, P. M. Sarosi, M. F. Henry, Investigation of creep deformation mechanisms at intermediate temperatures in Rene 88DT, Acta Materialia, 2005, 53: 3041.

[8] F. P. E. Dunne, A. J. Wilkinson, R. Allen, Experimental and computational studies of low cycle fatigue crack nucleation in a polycrystal, International Journal of Plasticity, 2007; 23:273.

[9] James Coakley, David Dye, Hector Basoalto, Creep and creep modeling of a multimodal nickel-base superalloy, Acta Materialia, 2011, 59: 854.

[10] S. Karthikeyan, R. R. Unocic, P. M. Sarosi, Modeling microtwinning during creep in Ni-based superalloys, Scripta Materialia, 2006, 54: 1157.

[11] A. Manonukul, F. P. E. Dunne, D. Knowles, Physically-based model for creep in nickel-base superalloy C263 both above and below the gamma solvus, Acta Materialia, 2002, 50: 2917.

[12] H. Gleiter, E. Hornbogen, Precipitation hardening by coherent particles, Material Science and Engineering, 1968, 2: 285.

[13] E. Nembach, G. Neite, Precipitation hardening of superalloys by ordered $\gamma^{\prime}$ particles, Progress in Materials Science, 1985, 29: 177.

[14] B. Reppich, P. Schepp, G. Wehner, Some new aspects concerning particle hardening mechanisms in $\gamma^{\prime}$ precipitating nickel-base alloys-II Experiments, Acta Materialia, 1982, 30: 95.

[15] P. Caron, T. Khan, P. Veyssiere, On precipitate shearing by superlattice stacking faults in superalloys, Philosophical Magazine A, 1988, 57:859.

[16] L. M. Brown and R. K. Ham, Strengthening Methods in Crystals, Applied Science Publishers, London, 1971.

[17] W. Huther, B. Reppich, Order hardening of $\mathrm{MgO}$ by large precipitated volume fractions of spinel particles, Materials Science and Engineering, 1979, 39:247.

[18] R. Reed, The superalloys fundamentals and applications, Cambridge University Press, 2006, 
74-86.

[19] M. P. Jackson, R. C. Reed, Heat treatment of Udimet 720Li: the effect of microstructure on properties, Materials Science and Engineering A, 259(1999), 85-97.

[20] G. B. Gibbs, Thermodynamic analysis of dislocation glide controlled by dispersed local obstacles, Materials Science and Engineering, 1969, 4:313.

[21] A. V. Granato, Entropy factors for thermally activated unpinning of dislocations, Journal of Applied Physics, 1964, 35: 2732.

[22] Hull. D, Introduction to Dislocations, $2^{\text {nd }}$ ed. UK: Pergamon Press, 1975. 